\title{
El sistema de predicción de la calidad del aire en AEMET: montaje, postproceso e índices
}

\author{
Isabel Martínez Marco' ${ }^{1}$ (imartinezm@aemet.es) \\ Alberto Cansado Auría ${ }^{1}$ (acansadoa@aemet.es) \\ María Allué Camacho' ${ }^{1}$ malluec@aemet.es) \\ Coralina Hernández Trujillo ${ }^{1}$ (chernandezt@aemet.es)
}

${ }^{1}$ AEMET / Departamento de Desarrollo y Aplicaciones / Área de Aplicaciones

\begin{abstract}
RESUMEN
La Agencia Estatal de Meteorología proporciona información sobre la composición química de la atmósfera mediante un sistema de predicción de la calidad del aire que suministra predicciones operativas de diferentes especies químicas, como son el ozono $\left(\mathrm{O}_{3}\right)$, el dióxido de nitrógeno $\left(\mathrm{NO}_{2}\right)$, el monóxido de nitrógeno (NO), el monóxido de carbono (CO) o el dióxido de azufre $\left(\mathrm{SO}_{2}\right)$, y material particulado de tamaños menores de 10 micras y menores de 2,5 micras, el PM10 y el PM2.5, sobre la península ibérica y Baleares. El sistema de predicción se basa en el modelo de transporte químico MOCAGE (Modèle de Chimie Atmosphérique de Grande Échelle), desarrollado por Météo-France, y utiliza el inventario de emisiones europeo TNO-MACC3 con base en las emisiones de 2011.
\end{abstract}

Los resultados de las predicciones en superficie de las especies citadas son publicados en la página web de AEMET (http://www.aemet.es/es/eltiempo/prediccion/calidad_del_aire). Las concentraciones aparecen expresadas en $\mu \mathrm{g} / \mathrm{m}^{3}$ y el contenido total de la columna de $\mathrm{O}_{3}$ en unidades Dobson. Sin embargo, este tipo de información puede ser muy técnica para el usuario y, por ello, se ha desarrollado un índice diario de calidad del aire de cada componente químico y un índice diario global. Por último, para mejorar las salidas del modelo en los puntos de observación se están desarrollando diferentes técnicas de postproceso para corrección del sesgo como el método Hybrid Forecast.

PALABRAS CLAVE: calidad del aire; modelización; composición química.

\section{INTRODUCCIÓN}

La composición química de la atmósfera terrestre está continuamente en evolución debido a los cambios en las emisiones (tanto naturales como antropogénicas) así como a la variabilidad climática natural (El Niño, etc.). Durante los últimos decenios, las autoridades sanitarias y relacionadas con el medio ambiente, junto a los medios de comunicación y el público en general, han mostrado un creciente interés por la contaminación atmosférica producida por actividades antropogénicas y sus consecuencias en la salud de las personas y de los ecosistemas. Los servicios meteorológicos, entre otras instituciones, han asumido el papel de suministrador de esta información utilizando para ello como herramienta los modelos de transporte químico. En este artículo se expondrán, en primer lugar, las características del modelo de transporte químico MOCAGE y su configuración en el sistema de predicción de la calidad del aire en AEMET. A continuación, se mostrarán las salidas de dicho sistema como los índices diarios de calidad del aire utilizados en AEMET. Y por último, se explicará el método de postproceso Hybrid-Forecast elegido en AEMET para aproximar los valores previstos por el modelo MOCAGE al valor de la observación en un punto y el trabajo futuro para mejorar el actual sistema de predicción de la calidad del aire. 


\section{MODELO DE TRANSPORTE QUÍMICO EN AEMET: MODELO MOCAGE}

El modelo de transporte químico utilizado en AEMET es el modelo francés MOCAGE (MOdèle de Chimie Atmosphérique à Grande Echelle) (JossE et al., 2004). MOCAGE es un modelo tridimensional global que puede funcionar con hasta tres dominios anidados adicionales, lo cual permite que se pueda ejecutar al mismo tiempo para varios dominios, cada vez más reducidos pero con una mayor resolución espacial. Este modelo proporciona simulaciones numéricas de las interacciones entre los procesos dinámicos, físicos y químicos en la troposfera y en la estratosfera, tanto para gases como para aerosoles. Tiene una resolución vertical de 47 niveles híbridos que se extienden desde la superficie hasta $0,1 \mathrm{hPa}$ (aproximadamente $60 \mathrm{~km}$ ), con 8 niveles por debajo de $2 \mathrm{~km}$, siendo el espesor de la capa más cercana al suelo de 40 metros.

Las principales características de MOCAGE son las siguientes:

- Su dominio vertical se extiende desde el suelo hasta la estratosfera $(\sim 5 \mathrm{hPa})$. De esta forma es posible simular fenómenos como la formación y evolución de los procesos de adelgazamiento de la capa de ozono (el conocido como agujero de ozono) sobre la Antártida al comienzo de cada primavera austral.

- Al ser un modelo global genera sus propias condiciones de contorno, lo que evita incoherencias entre diferentes modelos.

- MOCAGE funciona en AEMET como un modelo off-line. La dinámica está gobernada por predicciones meteorológicas de modelos de predicción numérica del tiempo (IFS/ECMWF, ARPEGE y HIRLAM) que MOCAGE utiliza como datos de entrada. En breve se sustituirá el modelo HIRLAM por el modelo HARMONIE.

- Posibilidad de asimilación variacional de datos: 3DVAR y 4DVAR. Actualmente no se utiliza la asimilación de datos.

- Esquema de advección semilagrangiana (WILLIAMSON y RASCH, 1989).

- Difusión turbulenta de Louis (Louss, 1979).

- Esquemas de convección de Tiedtke o de Kain-Fritsch-Bechtold (BECHTOLD et al., 2001).

- Deposición seca de Wesely (Wesely, 1989).

- Tratamiento separado de la deposición húmeda para precipitación convectiva y de gran escala.

El esquema químico utilizado, denominado RACMOBUS, es una fusión del esquema de química troposférica RACM (STOCKWELl et al., 1997) y del esquema de química estratosférica REPROBUS (LefÈvRE et al., 1994).

Es un modelo que tiene una resolución vertical muy buena (47 niveles híbridos) comparada con los estándares de los modelos de transporte químico. Posee más de 20 niveles en la estratosfera y alrededor de 7 dentro de la capa límite planetaria. Esto, junto al esquema químico utilizado, le permite una descripción correcta de procesos que se producen tanto próximos a la superficie como a nivel de la estratosfera.

En cuanto a las emisiones, MOCAGE puede utilizar diferentes inventarios de emisiones dependiendo del dominio espacial (global, regional o alta resolución) en el que nos encontremos. Las emisiones introducen incertidumbre en los resultados obtenidos por los modelos de transporte químico, por lo que estas deben ser elegidas en función de su exactitud y precisión y lo más actualizadas posible. 


\subsection{La configuración operativa de MOCAGE en AEMET}

La configuración operativa en AEMET consta de un dominio global a $2^{\circ}$ de resolución horizontal sobre el que se anida un dominio europeo a $0,5^{\circ}$ y finalmente un dominio peninsular a $0,1^{\circ}$ (fig. 1 ). Las islas Canarias no están todavía incluidas en el dominio de mayor resolución debido a la falta de un inventario de emisiones de alta resolución.

a)

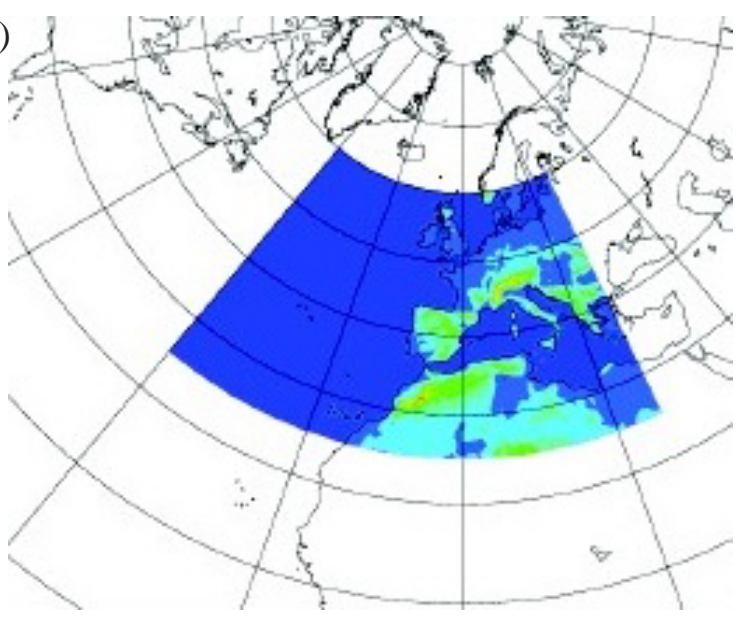

b)

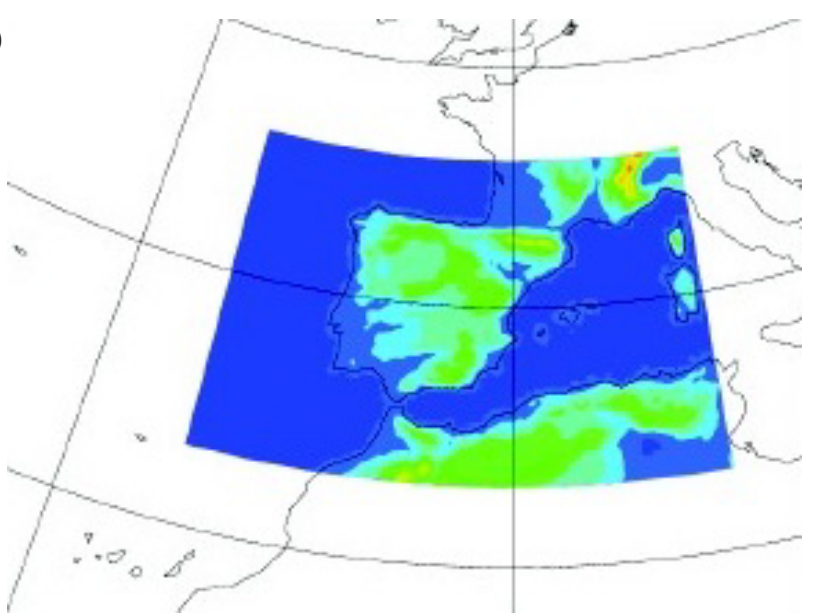

Figura 1. Dominios de la configuración operativa de MOCAGE en AEMET: a) europeo $\left(0,5^{\circ}\right)$ y b) peninsular $\left(0,1^{\circ}\right)$. Al modelo global a $2^{\circ}$ se anida el continental a $0,5^{\circ}$. Al continental se anida el peninsular a $0,1^{\circ}$.

Los forzamientos meteorológicos globales provienen del modelo IFS del Centro Europeo de Predicción a Plazo Medio (ECMWF) y de ARPEGE de Météo-France (superficiales). Los forzamientos para los dominios de $0,5^{\circ}$ y $0,1^{\circ}$, tanto en superficie como en altura, provienen del modelo HIRLAM HNR de AEMET de $0,05^{\circ}$ para el dominio peninsular $\left(0,1^{\circ}\right)$ y del HIRLAM ONR de AEMET de $0,16^{\circ}$ para el dominio europeo $\left(0,5^{\circ}\right)$. Las emisiones utilizadas sobre tierra y sobre mar en los dominios europeo y peninsular son las generadas por la empresa holandesa TNO para el proyecto MACC3 con una resolución nativa de $0,125^{\circ} \times 0,0625^{\circ}($ KUENEN et al., 2014).

Los forzamientos meteorológicos son información de viento, temperatura, humedad en todos los niveles y de presión en superficie. Esta información genera ficheros de forzamientos en altura cada tres horas que constituyen datos de entrada de MOCAGE. Además se incluye información de parámetros de superficie en los ficheros diarios de forzamientos superficiales que contienen datos como el albedo, características del suelo (espesor del suelo, proporción de arena o arcilla, ...), tipo de vegetación dominante o rugosidad entre otros. Adicionalmente son necesarias las velocidades de deposición de las especies consideradas, que son calculadas en el software que construye los ficheros de entrada con la información de superficie y son incluidas en estos mismos ficheros. Igualmente, las emisiones válidas para el día son generadas y añadidas al fichero que contiene los forzamientos superficiales.

Desde el mes de noviembre de 2011, MOCAGE se ejecuta operativamente en AEMET dos veces al día, a las 00 UTC con un alcance de predicción de 24 horas y a las 12 UTC con un alcance de 36 horas.

En un futuro próximo, se reemplazará el modelo HIRLAM HNR de $0,05^{\circ}$ por el modelo HARMONIE de $2,5 \mathrm{~km}$ de resolución horizontal y el modelo HIRLAM ONR de $0,16^{\circ}$ por el modelo del ECMWF con una resolución horizontal de alrededor de $9 \mathrm{~km}$. Se lanzarán dos pasadas al día, a las 00 y 12 UTC, con un alcance de 48 horas. 


\subsection{Predicciones obtenidas del modelo MOCAGE en AEMET}

Se publican las salidas de las predicciones en superficie de $\mathrm{O}_{3}, \mathrm{NO}_{2}, \mathrm{NO}, \mathrm{SO}_{2}, \mathrm{CO}, \mathrm{PM} 10$ y PM2.5 expresadas en $\mu \mathrm{g} / \mathrm{m}^{3}$ y el contenido total de la columna de $\mathrm{O}_{3}$ en unidades Dobson (http://www.aemet.es/es/ eltiempo/prediccion/calidad_del_aire).
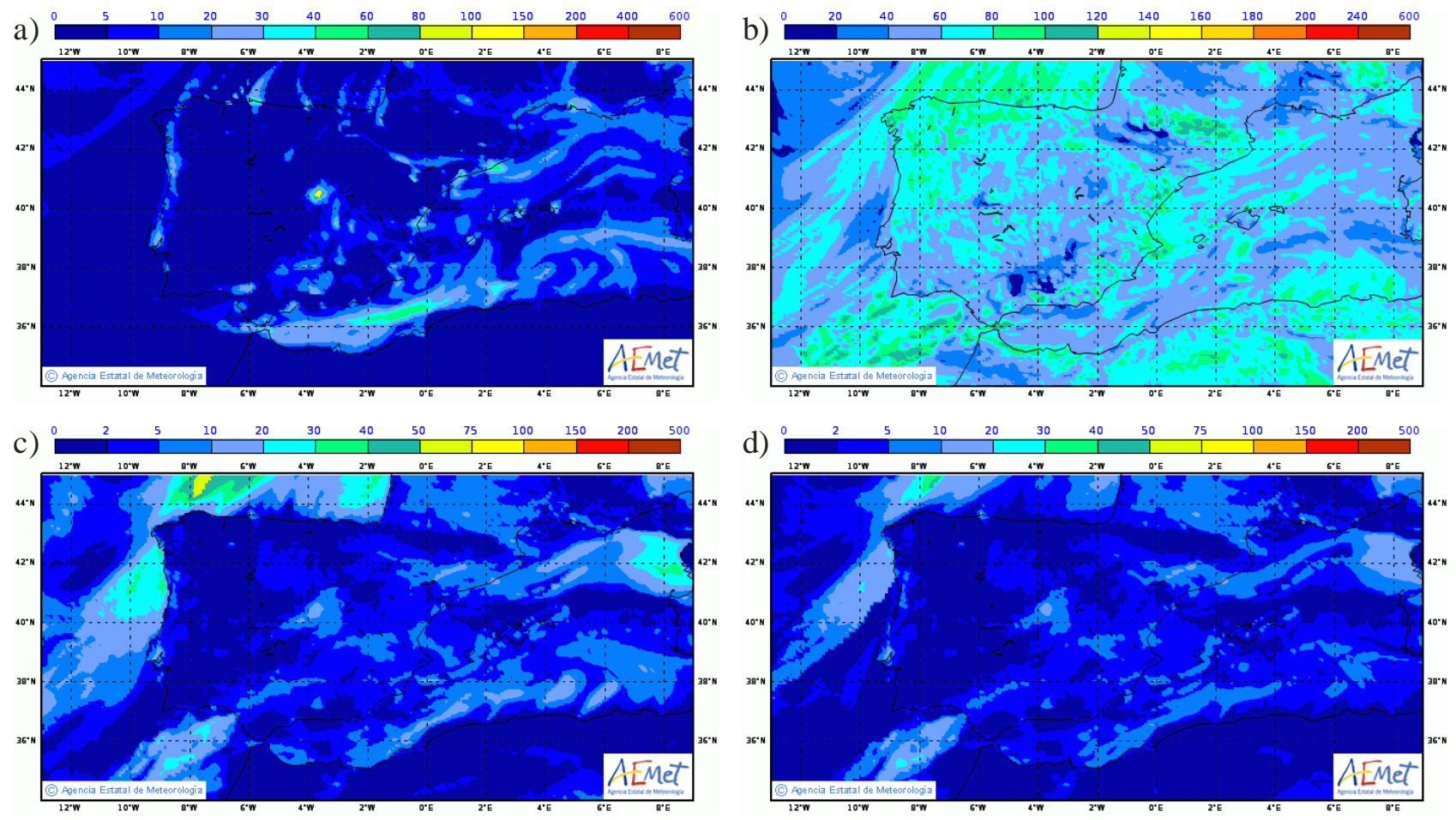

Figura 2. Concentración prevista en superficie en $\mu \mathrm{g} / \mathrm{m}^{3}$ : a) de $\mathrm{NO}_{2}$, b) de $\mathrm{O}_{3}$, c) de PM10 y d) de PM2.5, tal y como se publica en la página web externa de AEMET.

En la figura 2 se presenta un ejemplo de salidas de predicción de calidad del aire en AEMET. En este caso es una predicción a 12 horas del día 22 de noviembre de 2018 de la concentración prevista de $\mathrm{NO}_{2}, \mathrm{O}_{3}, \mathrm{PM} 10$ y PM2.5 en superficie. La paleta de colores de cada contaminante ha sido elegida teniendo en cuenta los colores correspondientes del índice europeo de calidad del aire de cada uno de los componentes químicos, aproximadamente, ya que los valores previstos son valores instantáneos cada hora.

Puesto que este tipo de información puede ser muy técnica y compleja, se ha desarrollado un índice diario de calidad del aire para cada componente químico y un índice diario global.

\section{3. ÍNDICES DIARIOS DE CALIDAD DEL AIRE}

Un índice de calidad del aire es un valor adimensional, calculado a partir de la información procedente de las directivas vigentes relacionadas con los distintos contaminantes atmosféricos, con el que se pretende conseguir hacer un resumen sobre el estado de la calidad del aire, facilitando al público la comprensión e interpretación de los valores cuantitativos de los diferentes contaminantes atmosféricos.

Se ha utilizado una metodología basada en el índice europeo de calidad del aire, desarrollado por la Agencia Europea de Medio Ambiente en base a las directivas europeas vigentes sobre calidad del aire, aunque se ha adaptado para proporcionar valores diarios, en lugar de valores horarios (http://www.eea.europa.eu/themes/ air/air-quality-index/index) (figura 3). 


$\begin{array}{cccccccl} & \begin{array}{c}\text { Índice Europeo } \\ \text { medias }\left(\mu \mathrm{g} / \mathrm{m}^{3}\right)\end{array} & \begin{array}{c}\mathbf{O}_{3} \\ \text { móvil } 8 \mathrm{~h}\end{array} & \begin{array}{c}\mathbf{N O}_{2} \\ 1 \mathrm{~h}\end{array} & \begin{array}{c}\mathbf{S O}_{2} \\ 1 \mathrm{~h}\end{array} & \begin{array}{c}\text { PM10 } \\ \text { móvil 24h }\end{array} & \begin{array}{c}\text { PM2.5 } \\ \text { móvil } 24 \mathrm{~h}\end{array} & \\ \text { Muy Buena } & 1 & 0-80 & 0-40 & 0-100 & 0-20 & 0-10 & \\ \text { Buena } & 2 & 80-120 & 40-100 & 100-200 & 20-35 & 10-20 & \text { Figura 3. } \\ \text { Moderada } & 3 & 120-180 & 100-200 & 200-350 & 35-50 & 20-25 & \text { Esquema del índice } \\ \text { Mala } & 4 & 180-240 & 200-400 & 350-500 & 50-100 & 25-50 & \text { diario previsto utilizado } \\ \text { Muy Mala } & 5 & >240 & >400 & >500 & >100 & >50 & \text { por AEMET. }\end{array}$

El índice se calcula a partir de los valores previstos en puntos de rejilla por el modelo MOCAGE y se presenta en forma de mapas (figura 4). Se calcula un índice parcial para el ozono $\left(\mathrm{O}_{3}\right)$, el dióxido de nitrógeno $\left(\mathrm{NO}_{2}\right)$, el dióxido de azufre $\left(\mathrm{SO}_{2}\right)$ y el material particulado PM10 y PM2.5 con un tratamiento diferenciado para cada especie:

- $\mathbf{N O}_{2}$ y $\mathbf{S O}_{2}$ : el índice viene determinado por el valor máximo entre los 24 valores horarios disponibles para un día.

- $\mathbf{O}_{3}$ : para cada hora del día se calcula la concentración media de las últimas ocho horas y el valor máximo de estas medias determina el valor del índice diario.

- PM10 y PM2.5: para cada hora del día se calcula la concentración media de las últimas 24 horas y el índice se define como el valor máximo de dichas medias móviles.

Finalmente, para generar el índice diario global previsto, que integra la información de todos los contaminantes, se selecciona el valor máximo de los índices parciales en cada punto de rejilla.

Figura 4.

a) Índice diario previsto global e índices diarios previstos para

b) $\mathrm{NO}_{2}$, c) $\mathrm{PM} 2.5$,

d) $\mathrm{PM} 10$, e) $\mathrm{SO}_{2} \mathrm{y}$

f) $\mathrm{O}_{3}$ para el martes 4 de septiembre de 2018.

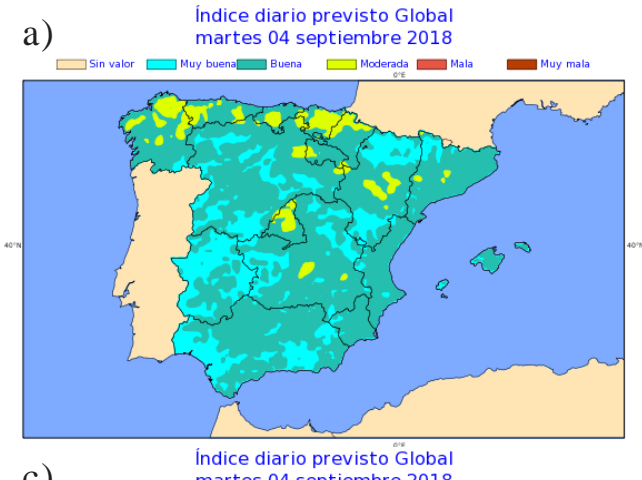

c)
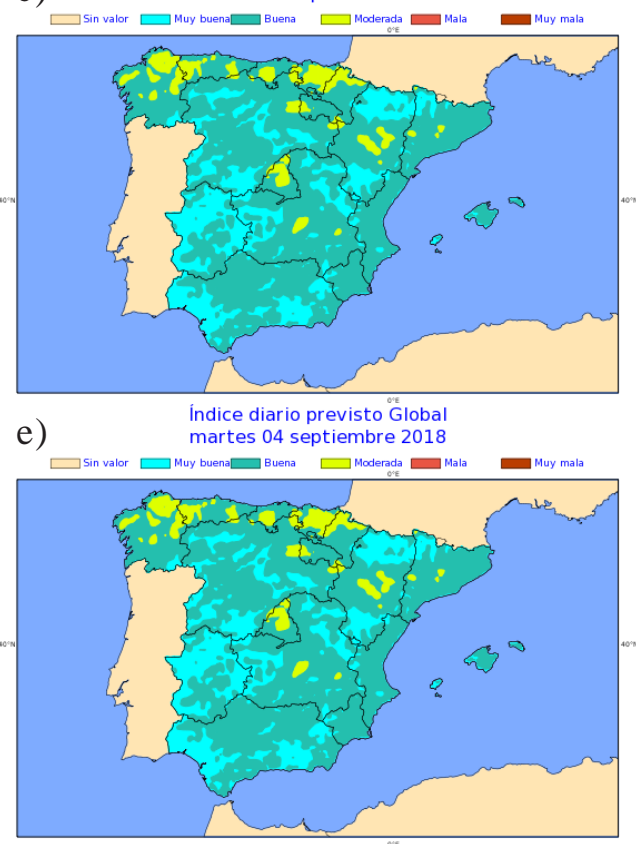

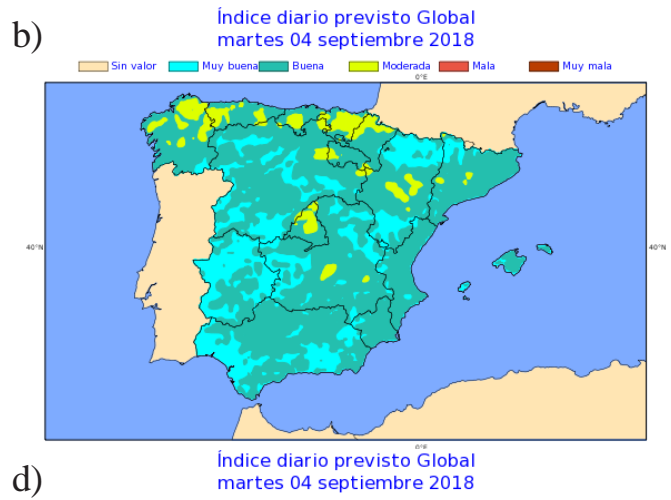

d)

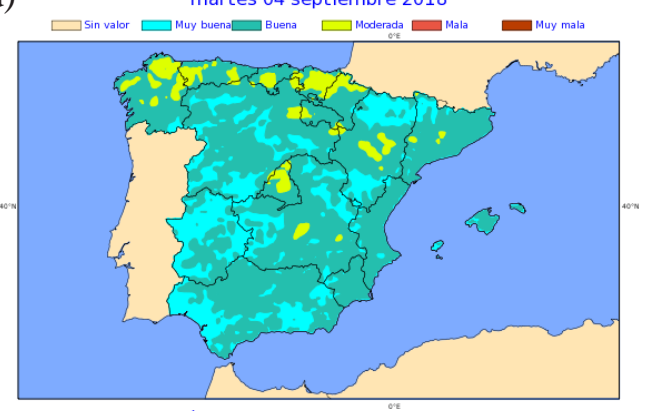

f)

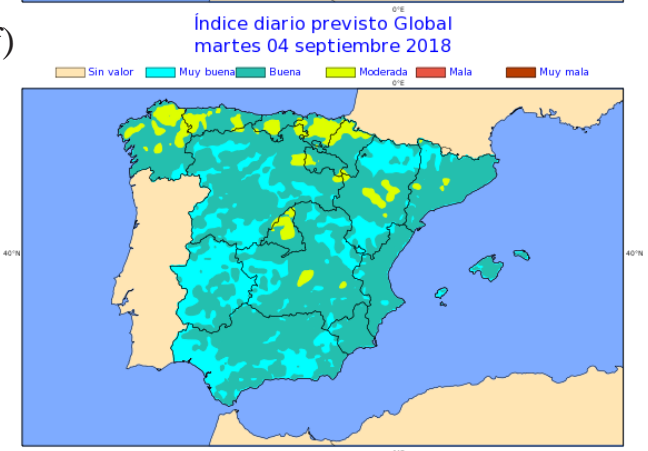


Se debe tener en cuenta que este índice se elabora a partir de la salida directa del modelo MOCAGE y por tanto adolece de las mismas limitaciones que presenta este. Los mapas son representativos de fenómenos a gran escala y pierden precisión conforme descienden a nivel local. Deben ser interpretados como tendencias y no como valores absolutos y pueden así contribuir a conocer mejor la predicción en términos cualitativos.

En general, la calidad de las predicciones del índice depende del contaminante que estemos considerando y, en algunos casos, de la estación del año en la que nos encontremos (HERNÁNDEZ-TruJiLlo et al., 2018).

\section{POSTPROCESO: MÉTODO HYBRID-FORECAST}

El estado de arte de los modelos de transporte químico no permite el uso directo de sus salidas en los puntos de observación. Para aproximar sus valores a los valores medidos, se utilizan métodos de postproceso como el MOS (Model Output Statistics) o la técnica llamada Hybrid Forecast (KANG et al., 2008), que utilizan las observaciones disponibles para corregir los valores previstos por el modelo.

La técnica Hybrid Forecast asume que el valor de la predicción en un sitio en un tiempo determinado es igual al valor de la salida del modelo más un residuo que se calcula como la diferencia entre la observación y el modelo 24 horas antes. Esta técnica es muy útil para contaminantes con sesgos significativos y alta homogeneidad espacial. Los mayores beneficios se observan en $\mathrm{el}_{3}$ y en el PM10.

En tiempo real, el problema de este método es el error en las observaciones. Para mitigar este problema se pueden utilizar técnicas que suavicen cualquier valor extremo. A su vez, esto puede provocar que no se reproduzca una superación real. Entonces, por ejemplo, el valor de la predicción en un punto se puede calcular como la mitad del residuo anterior más la mitad de la mediana de los residuos en todos los sitios con la misma clasificación (fondo, rural, suburbana y urbana). De esta manera se minimizan los posibles errores de una estación particular. En caso de rápidos cambios, se recomienda volver a recalcular este postproceso para incluir dicho cambio.

Utilizando esta técnica, hemos calculado los valores previstos de los diferentes componentes químicos en las estaciones de medida de calidad del aire que existen sobre la Península y Baleares, eliminando el sesgo y mejorando los diferentes valores estadísticos utilizados en la verificación del modelo MOCAGE. También nos permitirá calcular los índices horarios de calidad del aire sobre dichos puntos.

\section{TRABAJO FUTURO}

\subsection{Postproceso de las salidas del modelo MOCAGE con el método MOS (Model Output Statistics)}

Consiste en regresiones lineales múltiples entre una variable dependiente y un conjunto de variables independientes. La variable dependiente es la medida de la concentración del componente químico en las estaciones de medida en el día siguiente $(\mathrm{D}+1)$ al que se realiza el postproceso. Las variables independientes son las salidas previstas de esas mismas concentraciones proporcionadas por el modelo MOCAGE, las salidas previstas de variables meteorológicas proporcionadas por el modelo HARMONIE-AROME o el modelo del ECMWF, las salidas previstas de gases precursores o las medidas observadas de esas concentraciones en el día $\mathrm{D}$, etc. Se establecerán varias configuraciones y se verificará cuál da mejores resultados.

Es necesario utilizar un periodo de uno o dos años en tiempo pasado para entrenar la herramienta. También, dependiendo del contaminante, puede ser interesante separar el entrenamiento por estaciones climáticas (verano, otoño, invierno y primavera o agrupadas en meses fríos y cálidos) y por tipos de estaciones de medida: rural, suburbana y urbana.

Este tipo de postproceso, que mejora las desviaciones del modelo de predicción de calidad del aire del valor de las observaciones y minimiza los errores, suele reducir los valores de los extremos. Para estudiar su 
capacidad de predicción de episodios de alta concentración, se puede calcular el porcentaje de falsas alarmas y de alarmas no previstas. De esta manera, se elegirá la configuración que también mejore la predicción de este tipo de episodios.

Además, se estudiarán diferentes opciones para optimizar la predicción de superaciones de cada uno de los componentes químicos mencionados. Por ejemplo, realizar estas regresiones múltiples solo con los datos de valores de concentración más altos, eliminando los valores menores y dando de esta manera un mayor peso a esas situaciones.

\subsection{Métodos de «Downscaling»}

Se tratará de aumentar la resolución horizontal del modelo MOCAGE incorporando diferentes fuentes de datos tales como observaciones in situ o de satélite, inventarios de emisiones de alta resolución, emisiones biogénicas, etc.

\section{AGRADECIMIENTOS}

A Météo-France por ceder el modelo MOCAGE a AEMET a través de un acuerdo de colaboración entre ambas instituciones, tanto para investigación como para aplicaciones operacionales en el campo medioambiental.

\section{REFERENCIAS}

Bechtold, P., Bazile, E., Guichard, F., Mascart, P. y Richard, E., 2001. A mass flux convection scheme for regional and global models. Quart. J. Roy. Meteor. Soc., 127, 869-886.

Josse, B., Simon, P. y Peuch, V.-H., 2004. Rn-222 global simulations with the multiscale CTM MOCAGE. Tellus, 56B, 339-356.

Kang, D., Mathur, R., RaO, S. T. y Shaocai, Y., 2008. Bias adjustment techniques for improving ozone air quality forecasts. J. Geophys. Res., 113 (D23308).

Kuenen, J.J.P., VisschediJK, A. J.H., Jozwicka, M. y Denier van der Gon, H. A.C., 2014. TNO-MACC_II emission inventory; a multi-year (2003-2009) consistent high-resolution European emission inventory for air quality modelling. Atmos. Chem. Phys., 14, 10963-10976. doi: 10.5194/acp-14-10963-2014.

Hernández-Trujillo, C., Cansado, A. y Martínez, I., 2018. Verificación de las predicciones del modelo MOCAGE utilizando el índice europeo de calidad del aire. Acta de las Jornadas Científicas de la Asociación Meteorológica Española, 35, 322-336.

Lefèvre, F., Brasseur, G. P., Folkins, I., Smith, A. K. y Simon, P., 2004. Chemistry of the 1991-1992 stratospheric winter: three-dimensional model simulations. J. Geophys. Res., 99 (D4), 8183-8195.

Louis, J.F., 1979. A parametric model of vertical eddy fluxes in the atmosphere. B. Layer Meteor., 17 , 197-202.

STOCKwEll, W. R. et al., 1997. Anew mechanism for regional atmospheric chemistry modelling. J. Geophys. Res., 102, 25847-25879.

Williamson, D. L. y Rash, P. J., 1997. Two-dimensional semi-Lagrangian transport with shape preserving interpolation. Mon. Wea. Rev., 117, 102-129.

WeSEly, M.L., 1989. Parameterization of surface resistance to gaseous dry deposition in regional numerical models. Atmos. Env., 16, 1293-1304. 
